\title{
STABILIZATION OF ADIABATIC RIGID BODY ROTATION BY DISSIPATION*
}

\author{
BY
}

\author{
M. A. BOUDOURIDES
}

Democritus University of Thrace, Xanthi, Greece

\begin{abstract}
We consider the adiabatic tangential flow of an incompressible Newtonian fluid with temperature-dependent viscosity between two coaxial cylinders, one of which is rotating, while the other is kept stress-free. We show that every classical solution approaches, as time goes to infinity, a uniform rigid body rotation at fixed temperature, thus establishing the stabilizing role of dissipation. The proof is based on a priori estimates, obtained with the help of certain identities for solutions of the governing equations.
\end{abstract}

1. Introduction. The intent of this article is to contribute in the investigation of the stabilizing effect that the temperature dependence of viscosity induces to certain thermomechanical processes. In fact, we test the stabilizing effect of dissipation in the case of an adiabatic annular flow of an incompressible Newtonian fluid with temperature-dependent viscosity between two coaxial cylinders, one of which is rotating, while the other is kept stress-free.

All the previous works on the stabilization of thermomechanical processes ([3][6]) were focused on the case of an adiabatic rectilinear shearing between two parallel plates caused by various external agencies. Here, we are concerned with a tangential annular flow sustained by the uniform rotation of a rigid cylinder, where the fluid is also restricted by a coaxial cylindrical stress-free interface. Needless to say, such a tangential flow approximates better than rectilinear shearing the torsional tests under which the material response is experimentally determined. We should note that identical equations govern the behavior of a solid material, exhibiting strain rate sensitivity and thermal softening but no strain hardening, in the plastic region. In both cases, the only dissipative mechanism present is viscosity varying with temperature.

The flow region of our problem is the annulus between two coaxial cylinders, a rigid one at $r=1$, rotating uniformly around the axis with angular velocity $w=1$, and a frictionless (stress-free) second one at $r=a$. We will assume that $0<a<1$. Then an incompressible Newtonian fluid occupying this region will move in a circular pattern with an angular velocity $w(r, t)$ (where $r w=v$ is the tangential velocity) and under a pressure $p(r, t)$ balancing the centrifugal force. Under adiabatic conditions

*Received September 11, 1986. 
and upon identifying internal energy with temperature $\theta(r, t)$, the evolution of the fields $w(r, t), p(r, t)$, and $\theta(r, t)$ (for unit density) is governed by the following balance laws of momentum and energy (see [1] for their derivation):

$$
\begin{aligned}
r w^{2} & =p_{r}, \\
w_{t} & =\frac{1}{r^{3}}\left(r^{2} \sigma\right)_{r}, \quad a<r<1, t>0 . \\
\theta_{t} & =\sigma r w_{r} .
\end{aligned}
$$

Above, $\sigma(r, t)$ is the shear stress, for which we adopt the following Newtonian constitutive relation:

$$
\sigma=\mu(\theta) r w_{r} .
$$

We assume that the viscosity $\mu$ is a decreasing function of temperature of the form

$$
\mu(\theta)=\theta^{-\gamma}, \quad \gamma>0 .
$$

Such a temperature dependence of viscosity, in addition to being typical for liquids (cf. [2, p. 15]), is the most interesting case in the study of the asymptotic behavior of solutions of equations (1.1)-(1.4), as shall be seen below.

The system of equations (1.1)-(1.5) together with the boundary conditions

$$
\begin{array}{ll}
w=1, & r=1, \\
\sigma=0, & r=a,
\end{array} \quad t>0,
$$

(i.e. no-slip at $r=1$ and stress-free at $r=0$ ) and the initial conditions

$$
\begin{aligned}
w & =w_{0}, \quad a \leq r \leq 1, t=0, \\
\theta & =\theta_{0},
\end{aligned}
$$

that satisfy the compatibility conditions $w_{0}=1, r=1$, and $w_{0 r}=0, r=a$, will determine $w(r, t), p(r, t)$, and $\theta(r, t)$ on $[a, 1] \times[0, \infty)$.

From the rotation of the rigid boundary, energy is pumped into the system, and as the process is adiabatic, temperature will keep rising with time. Under assumption (1.5), $\mu(\theta)$ tends to zero as $\theta \rightarrow \infty$, and the asymptotic behavior of solutions of (1.2) becomes delicate. The question is whether the temperature increase will be uniform or whether it could localize, thus destabilizing the system. (For a solid, destabilization would be possibly manifested by shear band formation.)

From the point of view of analysis, the answer depends upon the outcome of the contest between the destabilizing effect of hyperbolicity of Eq. (1.3) and the stabilizing effect of dissipation in Eq. (1.2). However, under the adopted dependence of viscosity on temperature by (1.5), the analysis establishes that temperature increases in an "orderly" fashion to reach asymptotically a finite stationary distribution $\bar{\theta}(r)$, and angular velocity approaches asymptotically the uniform rigid body rotation $\bar{w}(r)=1$, preventing any development of singularities. In other words, the uniform rigid body rotation at fixed finite temperature distribution is the unique asymptotically stable solution of (1.1)-(1.9). More precisely, we will prove the following result. 
THEOREM. Let $\mu$ depend on $\theta$ as in (1.5). Assume that $w_{0} \in W^{2,2}(a, 1), \theta_{0} \in$ $W^{1,2}(a, 1), \theta_{0}(r)>0, a \leq r \leq 1$. Then there is a unique classical solution of (1.1)-(1.9) on $[a, 1] \times[0, \infty)$, and as $t \rightarrow \infty$,

$$
\begin{aligned}
\sigma(r, t) & =O\left(e^{-K t}\right), \\
w(r, t) & =1+O\left(e^{-K t}\right), \\
p(r, t) & =r^{2} / 2+O\left(e^{-K t}\right), \\
|\theta(r, t)| & \leq K, \\
\theta(r, t) & \rightarrow \bar{\theta}(r)>0,
\end{aligned}
$$

uniformly in $r$ on [a,1], for some positive constant $K$ depending solely on $a, \gamma, w_{0}$, and $\theta_{0}$.

2. Proof of Theorem. Let us assume that $(w(r, t), p(r, t), \theta(r, t))$ is a fixed classical solution of $(1.1)-(1.9)$ on $[a, 1] \times[0, \infty)$ such that $w(\cdot, t), w_{r}(\cdot, t), w_{t}(\cdot, t), w_{r r}(\cdot, t)$, $p(\cdot, t), p_{r}(\cdot, t), \theta(\cdot, t), \theta_{r}(\cdot, t)$ are all in $C^{0}\left([0, \infty) ; L^{2}(a, 1)\right)$, while $w_{r t}(\cdot, t)$ is in $C^{0}((0, \infty)$; $\left.L^{2}(a, 1)\right)$ and $w_{t t}(\cdot, t)$ is in $L_{\mathrm{loc}}^{2}\left((0, \infty) ; L^{2}(a, 1)\right)$.

We begin by deriving certain identities that will be used to establish the a priori estimates that will lead to the proof of the theorem.

LEMMA 2.1.

$$
\begin{gathered}
\int_{0}^{t} \int_{a}^{1} r^{3} w_{t}^{2} d r d \tau+\frac{1}{2} \int_{0}^{1} r^{3} \theta^{-\gamma} w_{r}^{2} d r+\frac{\gamma}{2} \int_{0}^{t} \int_{a}^{1} r^{5} \theta^{-2 \gamma-1} w_{r}^{4} d r d \tau \\
=\frac{1}{2} \int_{a}^{1} r^{3} \theta_{0}^{-\gamma} w_{0 r}^{2} d r \quad \quad t \geq 0, \\
\frac{1}{2} \frac{d}{d t} \int_{a}^{1} r^{3} w_{t}^{2} d r+\int_{a}^{1} r^{3} \theta^{-\gamma} w_{r t}^{2} d r=\gamma \int_{a}^{1} r^{2} \theta^{\gamma-1} \sigma^{3} w_{r t} d r, \quad t>0 .
\end{gathered}
$$

Proof. To show (2.1), after substituting $\sigma$ in (1.2) from (1.4) and (1.5), multiply by $r^{3} w_{t}$, integrate over $[a, 1] \times[0, t]$, and integrate by parts, using (1.3)-(1.7).

To show (2.2), after substituting $\sigma$ in (1.2) from (1.4) and (1.5), differentiate with respect to $t$ and then multiply by $r^{3} w_{t}$, integrate over $[a, 1]$, and integrate by parts, using (1.4)-(1.7).

The next lemma summarizes some useful relations obtained from the equations and the boundary conditions.

LEMMA 2.2. For $a \leq r \leq 1, t \geq 0$,

$$
\begin{gathered}
p(r, t)=\int_{a}^{r} \rho w^{2}(\rho, t) d \rho, \\
\theta^{1-\gamma}(r, t)-\theta_{0}^{1-\gamma}(r)=(1-\gamma) \int_{0}^{t} \sigma^{2}(r, \tau) d \tau, \\
r^{4} \theta^{-\gamma}(r, t) \theta_{r}(r, t)+\frac{4}{1-\gamma} r^{3} \theta^{1-\gamma}(r, t) \\
=r^{4} \theta_{0}^{-\gamma}(r) \theta_{0 r}(r)+\frac{4}{1-\gamma} r^{3} \theta_{0}^{1-\gamma}(r)+2 \int_{0}^{t} r^{5} \sigma(r, \tau) w_{t}(r, \tau) d \tau,
\end{gathered}
$$




$$
\begin{gathered}
r^{2} \sigma(r, t)=\int_{a}^{r} \rho^{3} w_{t}(\rho, t) d \rho, \\
r^{4} \sigma^{2}(r, t)=2 \int_{a}^{r} \rho^{5} \sigma(\rho, t) w_{t}(\rho, t) d \rho, \\
w(r, t)=1-\int_{r}^{1} w_{r}(\rho, t) d \rho .
\end{gathered}
$$

Proof. Integrate (1.1) to obtain (2.3). Substitute (1.4) and (1.5) into (1.3) to get

$$
\frac{1}{1-\gamma}\left(\theta^{1-\gamma}\right)_{t}=\sigma^{2}
$$

which integrated over $[0, t]$ yields (2.4). Multiply (2.9) by $r^{4}$, differentiate with respect to $r$, use (1.2), and then integrate over $[0, t]$ to obtain (2.5). Apply (1.7) to get (2.6), and combine with (1.2) to get (2.7). Finally, (2.8) follows from(1.6).

Now, we are in the position to establish the a priori estimates that will yield the proof of the theorem. In what follows, $K$ will stand for a generic constant which can be estimated from above solely in terms of $\gamma, a$, and upper bounds of the $W^{2,2}(a, 1)$ norm of $w_{0}$ and the $W^{1,2}(a, 1)$-norm of $\theta_{0}$.

The first estimate refers to the $L^{2}$-norm of $w_{t}$ and $\sigma$.

LEMMA 2.3. For $a \leq r \leq 1, t \geq 0$,

$$
\begin{gathered}
\int_{0}^{t} \int_{a}^{1} w_{t}^{2} d r d \tau \leq K, \\
\int_{0}^{t} \sigma^{2} d \tau \leq K, \\
\int_{a}^{1} \sigma^{2} d r \leq K .
\end{gathered}
$$

Proof. (2.10) is a direct consequence of (2.1). Applying the Cauchy-Schwarz inequality to (2.7) and using (2.10), we obtain (2.11). Finally, (1.4), (1.5), and the fact that $\theta(\cdot, t)$ is nondecreasing imply that

$$
\int_{a}^{1} \sigma^{2} d r \leq a^{-1}\left(\min _{[a, 1]} \theta_{0}\right)^{-\gamma} \int_{a}^{1} r^{3} \theta^{-\gamma} w_{r}^{2} d r
$$

from which, after using (2.1), we get (2.12).

The next lemma establishes that $\theta$ is bounded.

LEMMA 2.4. For $a \leq r \leq 1, t \geq 0$, it is

$$
\theta_{0}(r) \leq \theta(r, t) \leq K
$$

Proof. Use (2.4) and (2.11) to get the upper bound. Since $\theta(\cdot, t)$ is nondecreasing, we get the lower bound.

We now proceed to estimate the $L^{2}$-norm of $w_{t}$. 
LEMMA 2.5. For $t \geq 0$,

$$
\int_{a}^{1} w_{t}^{2} d r \leq K e^{-K t}
$$

Proof. Applying the Cauchy-Schwarz inequality to (2.2), we obtain, using (2.13),

$$
\frac{d}{d t} \int_{a}^{1} r^{3} w_{t}^{2} d r+K \int_{a}^{1} r^{3} w_{r t}^{2} d r \leq K \int_{a}^{1} \sigma^{6} d r
$$

Applying the Cauchy-Schwarz inequality to (2.7), we obtain, using (2.12),

$$
\int_{a}^{1} \sigma^{6} d r \leq\left(\max _{[a, 1]} \sigma^{2}\right)^{2} \int_{a}^{1} \sigma^{2} d r \leq K \int_{a}^{1} r^{3} w_{t}^{2} d r
$$

because of which $(2.15)$ yields

$$
\frac{d}{d t} \int_{a}^{1} r^{3} w_{t}^{2} d r \leq K \int_{a}^{1} r^{3} w_{t}^{2} d r
$$

Integrating the above relation over $[0, t]$ and using $(2.10)$, we get

$$
\int_{a}^{1} r^{3} w_{t}^{2} d r \leq K
$$

Now, applying the Cauchy-Schwarz inequality to (2.8) and then integrating over $[a, 1]$, we obtain

$$
\int_{a}^{1} r^{3} w_{t}^{2} d r \leq K \int_{a}^{1} r^{3} w_{r t}^{2} d r
$$

Again, the Cauchy-Schwarz inequality applied to (2.6) yields, on account of (1.4), (1.5), and (2.13),

$$
w_{r}^{2} \leq K \int_{a}^{1} r^{3} w_{t}^{2} d r
$$

i.e.,

Thus, setting

$$
\int_{a}^{1} \sigma^{6} d r \leq K\left(\int_{a}^{1} r^{3} w_{t}^{2} d r\right)^{3} .
$$

$$
\psi(t)=\int_{a}^{1} r^{3} w_{l}^{2} d r
$$

and combining (2.15) with (2.17) and (2.18), we obtain the integrodifferential inequality

$$
\frac{d \psi}{d t}+K_{1} \psi \leq K_{2} \psi^{3}
$$

where, by account of (2.10) and (2.16),

$$
\int_{0}^{t} \psi(\tau) d \tau \leq K_{0}
$$

and

$$
\psi(t) \leq K .
$$

Because of the last two inequalities, there exists a $t_{0} \geq 0$ such that

$$
\psi(t)<\left(K_{1} /\left(2 K_{2}\right)\right)^{1 / 2}, \quad t \geq t_{0} .
$$


Therefore, for $t \geq t_{0},(2.19)$ becomes

$$
\frac{d \psi}{d t} \leq(-1 / 2) K_{1} \psi
$$

Integrating the last differential inequality over $\left[t_{0}, t\right]$, we deduce

$$
\psi(t) \leq \psi\left(t_{0}\right) e^{\left(-K_{1} / 2\right)\left(t-t_{0}\right)}, \quad t \geq t_{0},
$$

and combining with (2.20), we obtain (2.14) for any $t \geq 0$.

Applying the Cauchy-Schwarz inequality to (2.7) and using (2.12) and (2.14), we arrive at

$$
\sigma^{2} \leq K e^{-K t}
$$

which proves $(1.10)$.

Using (1.4), (1.5), (2.13), and (1.10),

$$
\left|w_{r}\right| \leq K e^{-K t}
$$

and so $(2.8)$ yields

$$
|w-1| \leq K e^{-K t},
$$

i.e., (1.11) is valid, from which, on account of (2.3), we arrive at (1.12).

Applying the Cauchy-Schwarz inequality to (2.5) and using (2.13), (2.11), and (2.14), we obtain immediately (1.13).

Since, for every $a \leq r \leq 1, \theta(r, t)$ is a nondecreasing and bounded function of $t, \theta(r, t)$ converges as $t \rightarrow \infty$ to some $\bar{\theta}(r)<\infty$. Furthermore, by (1.13) and the Ascoli-Arzela theorem, the convergence is uniform in $r$, which proves (1.14).

Thus, the above a priori estimates establish the existence of a unique global solution of (1.1)-(1.9) by a routine procedure, first showing that a unique local solution exists on a maximal time interval and then using the a priori estimates to infer that this solution cannot escape in a finite time.

Acknowledgment. The author wishes to thank Constantine M. Dafermos for providing many stimulating discussions during the author's visit at Brown University in spring 1986.

\section{REFERENCES}

[1] G. K. Batchelor, An introduction to fluid dynamics, Cambridge University Press, London (1970)

[2] R. B. Bird, W. E. Stewart, and E. N. Lightfoot, Transport phenomena, Wiley, New York-London (1960)

[3] N. Charalambakis, Adiabatic shearing flow caused by time dependent inertial force, Quart. Appl. Math. 42, 275-280 (1984)

[4] C. M. Dafermos, Stabilizing effects of dissipation, in H. W. Knobloch and K. Schmitt (eds.), Equadiff 82, Lecture Notes in Math., vol. 1017, Springer-Verlag, 140-147 (1983)

[5] C. M. Dafermos and L. Hsiao, Adiabatic shearing of incompressible fluids with temperature dependent viscosity, Quart. Appl. Math. 41, 45-58 (1983)

[6] A. E. Tzavaras, Shearing of materials exhibiting thermal softening or temperature dependent viscosity, Quart. Appl. Math. 44, 1-12 (1986) 